\title{
Oil adjuvant elevates protection of rainbow trout (Oncorhynchus mykiss) following injection
} vaccination against Yersinia ruckeri

\author{
Rzgar M. Jaafar ${ }^{\mathrm{a}, \$}$, Jiwan K Chettri ${ }^{\mathrm{a}}$, Inger Dalsgaard ${ }^{\mathrm{b}}$, Azmi Al-Jubury ${ }^{\mathrm{a}}$, Per W. Kania ${ }^{\mathrm{a}}$, Jakob \\ Skov $^{\text {a }}$, Kurt Buchmann ${ }^{\mathrm{a}}$ \\ ${ }^{a}$ Department of Veterinary Disease Biology, Faculty of Health and Medical Sciences, University of \\ Copenhagen, Frederiksberg C, Denmark
}

${ }^{b}$ National Veterinary Institute, Technical University of Denmark, Frederiksberg C, Denmark

\begin{abstract}
Enteric redmouth disease (ERM) caused by the fish pathogen Yersinia ruckeri is a major threat to freshwater production of rainbow trout throughout all life stages. Injection vaccination of rainbow trout against $Y$. ruckeri infection has been shown to confer better protection compared to the traditionally applied immersion vaccination. It may be hypothesized, based on experience from other vaccines, that adjuvants may increase the protective level of ERM injection vaccines even more. Controlled comparative vaccination studies conducted to investigate effects of the oil adjuvant Montanide ${ }^{\mathrm{TM}}$ ISA 763 A VG when added to an experimental Y. ruckeri bacterin (containing both biotype 1 and 2 of serotype O1). A total of 1000 fish with mean weight $19 \mathrm{~g}$ was divided into five different groups (in duplicated tanks $2 \times 100$ fish per group) 1) nonvaccinated control fish (NonVac), 2) fish injected with a commercial vaccine (AquaVac ${ }^{\circledR}$ Relera $^{\mathrm{TM}}$ ) (ComVac), 3) fish injected with an experimental vaccine (ExpVac), 4) fish injected with an experimental vaccine + adjuvant (ExpVacAdj) and 5) fish injected with adjuvant alone (Adj). Injection of the experimental vaccine (both adjuvanted and non-adjuvanted) induced a significantly higher antibody (IgM) level, increased occurrence of $\operatorname{IgM}^{+}$cells in spleen tissue and significant up-regulation of several immune genes. Additional experiments using a higher challenge dosage suggested an immune enhancing effect of the adjuvant as the challenge produced 100\% mortality in the NonVac group, $60 \%$ mortality in both of ComVac and Adj groups and only 13 and $2.5 \%$ mortalities in the ExpVac and the ExpVacAdj groups, respectively.
\end{abstract}

\section{Keywords}

Rainbow trout; Yersinia ruckeri; Vaccination; Adjuvant

These authors have contributed equally to this work

${ }^{\$}$ Corresponding author. Tel: +45 35333137

E-mail address: rezgarhadad@ sund.ku.dk (Rzgar M. Jaafar). 